\title{
Flexible ECG circuit fabrication and application using vinyl cutting technique
}

\author{
Noor Syazwana Sahar ${ }^{1}$, Nurul Ashikin Abdul-Kadir ${ }^{2}$, Fauzan Khairi Che Harun ${ }^{3}$ \\ ${ }^{1}$ School of Biomedical Engineering and Health Sciences, Faculty of Engineering, \\ Universiti Teknologi Malaysia, Johor Bahru, Malaysia \\ ${ }^{2,3}$ School of Electrical Engineering, Faculty of Engineering, Universiti Teknologi Malaysia, Johor Bahru, Malaysia
}

\begin{tabular}{l} 
Article Info \\
\hline Article history: \\
Received Jan 11, 2020 \\
Revised Mar 30, 2020 \\
Accepted Apr 17, 2020 \\
\hline
\end{tabular}

\section{Keywords:}

Circuit fabrication

Conductive pattern

ECG

Flexible ECG circuit

Vinyl cutting

\begin{abstract}
The aim of this study is to prove the capability of vinyl cutting technique to cut the conductive traces of electronic circuit layout which used a copper tape (Copper tape 1181 from $3 \mathrm{M}$ ) on flexible substrate to replace the method of using nano-scale particle material. A wireless electrocardiography (ECG) circuit was integrated and fabricated on flexible substrate, namely a polyethylene terephthalate (PET) substrate by using vinyl cutting method to produce the conductive line traces. After that, the fabricated circuit is used for acquiring ECG signals from a patient simulator and human subjects to measure the performance differences and compatibility as a wearable device. In the data processing stage, ECG data were denoised using sym20 from Wavelet Transform tool provided by MATLAB. Then, Signal-to-noise-ratio (SNR) was calculated and used as the signal quality indicator. At the end of the study, flexible circuit performance was compared to MIT-BIH Arrhythmia database and it shows that there is no significance difference between both. In conclusion, vinyl cutting method shows a promising fabrication output on PET substrate as the performance of both flexible ECG circuit is comparable with rigid ECG circuit by a previous study.
\end{abstract}

This is an open access article under the CC BY-SA license.



\section{Corresponding Author:}

Fuazan Khairi Che Harun,

School of Electrical Engineering,

Faculty of Engineering,

Universiti Teknologi Malaysia,

81310 UTM Johor Bahru, Johor, Malaysia.

Email: fauzan@utm.my

\section{INTRODUCTION}

To date, the development of electronic devices with both excellent electrical conductivity and outstanding flexibility is an active and vibrant research field specifically in a production of flexible electronics devices such as flexible displays [1], flexible antenna arrays [2], radio-frequency identification (RFID) tags [3], and biomedical devices [4]. Important features of new electronic devices are low manufacturing costs, longer endurance time, recycling, lower energy consumption and higher efficiency.

Flexible electronic circuits can be generally fabricated by depositing the patterned conductive materials onto the flexible substrate via conventional vacuum deposition or printing processes. These technologies contribute to the potentially low-cost process but still there are many problems arouse which needed to be encountered and concerned, such as the effect of environmentally pollution and defective production which are the challenge [5]. On the other hand, Suikkola et al [6] have done a research on fabrication and characterization of stretchable interconnects for wearable electronics application. Their focus is to study the changes which occur on the traces that were screen printed with a stretchable silver-polymer 
composite ink on thermoplastic polyurethane (TPU). From the study, there are changes in resistance over strain. This behavior is not suitable to be used in a sensor's circuit because it would interrupt the accuracy and contribute noise to the electronic system.

On top of that, Eshkeiti [7]has done a research on screen printing of multilayered hybrid PCB on different substrates. He used PET, glass and paper as the flexible substrates in his study. Screen printing method and silver (Ag) flake ink (Electrodag 479SS) and an UV acrylic-based ink (Electrodag PF-455B) from Henkel were used as the metallization and dielectric layers, respectively. However, the same problem occurred of which the resistivity of the traces changed whenever there are bending or moving body's activity. It can be concluded that, there would be a problem of having loss continuity if nano-size conductive ink is used as the material to fabricate the conductive traces that may produce unstable conductivity.

Another research has mentioned the advantages and disadvantages of using inkjet printing for circuit fabrication [8]. This method is able to give a better resolution for conductive traces than screen printing method. However, there is a high possibility for the nozzles to clog because of the accumulation of the nanoparticles ink inside the cartridge. Besides that, the stability of the conductive traces printed by the method is less because of low concentration of nanoparticles ink. This issue leads to high resistance of conductive traces.

Extensive research and development are required to overcome these technical problems to improve the material printing technology up to the manufacturing process. For example, how to improve conductivity and resolution of printed circuits, improve adhesion between the printed materials and the substrates, further reduce the cost, and enrich choices of substrate materials. Thus, in this study, the focus is to use vinyl cutting method and copper tape as the conductive traces on flexible substrate. In addition, copper tape is used instead of nano-size conductive ink. Previously, vinyl cutting method has been used in fabrication process of electronic devices. However, the implementations were on different applications such as silicone devices [9] and flexible transparent free-form displays [10].

\section{RESEARCH METHOD}

This section discusses on the method used in this study to achieve the objectives of the study. The process flow of integrating ECG device onto the PET substrate and data collection is shown as in Figure 1. Earlier literature study and preliminary study could be found at [11-13]. The study was started by designing the ECG circuit. It was designed using EAGLE software by EAGLE Autodesk. Details on the circuit design and fabrication are discussed in section 2.1. The design was a single sided circuit layout. Next is the fabrication process on PET substrate using vinyl cutter machine and testing the circuit functionality. Last stage of this study was data collection and analysis which are discussed in section 2.2.

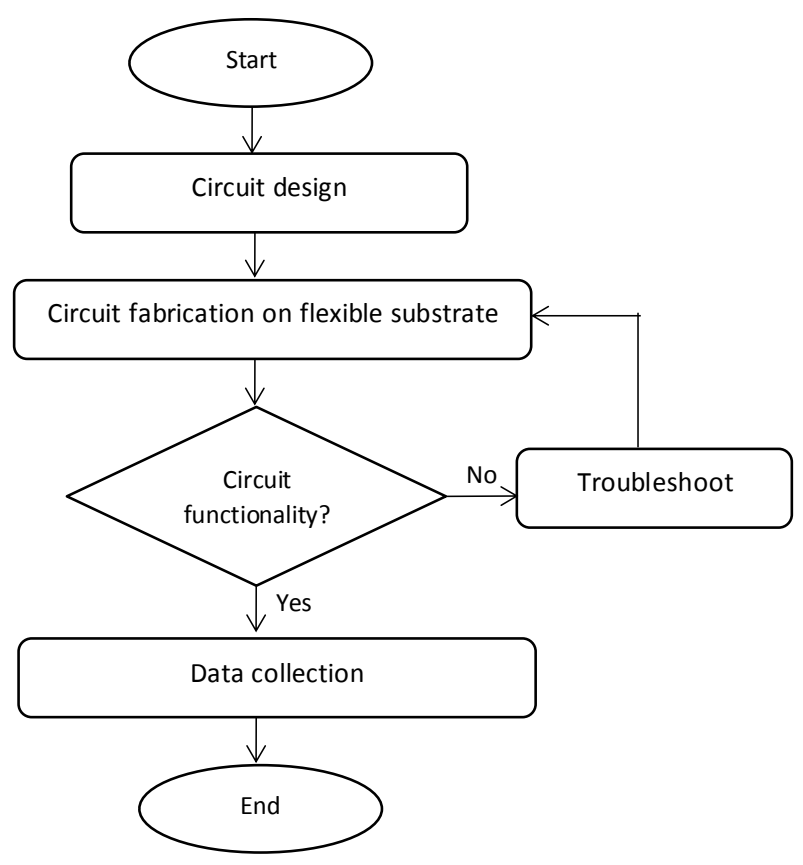

Figure 1. The study flow diagram 


\subsection{Circuit design and fabrication}

Before designing a circuit, component selection was done. First of all, in order to design a wireless ECG device, an efficient wireless protocol module and digital signal processing (DSP) chip for bio signal detection are needed to assure convenience to the end user. The developed device shall consider portability and wearability aspects. Bluetooth HC05 module is chosen as it is designed for transparent wireless serial connection setup. Thus, the communication is via serial communication which makes an easy way to communicate with a controller or a computer. This Bluetooth module also provides switching mode between master and slave modes; which means it is equipped with the capability to be used as a neither receiver nor transmitter. In this study, the Bluetooth is acted as a transmitter as it will send the data to the phone before further processing.

Meanwhile for DSP module, BMD101 integrated circuit (IC) from NeuroSky is used for acquiring an accurate bio signal detection and processing [13]. This IC has high performance of its analog front end (AFE) for bio signal detection range from microvolt level to millivolt level. This chip is built with high pass filter (HPF), low noise amplifier (LNA) and analogue digital converter (ADC) for the part of AFE. Most of the bio signal filtering are done in the digital domain, after the data leaves the ADC, it goes through the digital filter such as notch filter and low pass filter (LPF). Next is the ECG circuit design. Circuit design process has been done using EAGLE CADSOFT software. After completing the ECG circuit design, then, fabrication on PET substrate using vinyl cutter was run. To complete this study, equipments and materials used are as follow; vinyl cutter machine SummaCut D60R, Memmert oven, 3M Copper Foil Tape 1181, PDMS (Sylgard 184 Silicone Elastomer) and conductive Epoxy (CircuitWorks CW2400) from Chemtronics.

The process started with transferring the copper tape layer onto the PET substrate. The most suitable size for the copper tape and substrate to be used with vinyl cutter machine was according to A4 paper size. Once the copper tape has been attached to PET substrate, it is inserted into the vinyl cutter machine to make the traces according to the design. To cut the traces on the copper tape, appropriate knife pressure should be used to prevent the vinyl cutter from cutting through the substrate. Knife pressure used was $80 \mathrm{~g}$ with velocity of $50 \mathrm{~mm} / \mathrm{s}$. Next, when the cutting process has been completed, peel-off the unused copper tape to get the conductive traces according to the design we have set earlier using Eagle software. After that, the circuit is ready to be assembled with the electronic components.

In assembling the electronics component on the PET substrate, a silver conductive adhesive from CircuitWorks (CW2400 from Chemtronics) is used as the solder paste replacement. The reason it is used for flexible circuit fabrication is to replace solder paste which require a higher temperature for curing. In comparison of several conductive adhesives, this silver epoxy is having the lowest resistance $(0.5 \Omega)$ for each contact of mounting surface-mount device (SMD) component and provides mechanically stable contact [14]. This conductive epoxy comes with two parts: part A is epoxy and part B is the hardener. Equal amounts (1:1) by weight or volume of Part A and Part B were mixed thoroughly for 2-3 minutes and immediately applied on the substrate' surface. Then, the components were placed accordingly on the applied conductive epoxy. To get maximum conductivity and adhesion, it must be cured at $80{ }^{\circ} \mathrm{C}$ in 10 minutes. The curing time is following the suggestion provided on the datasheet.

Last step is polydimethylsiloxane (PDMS) encapsulation process. The encapsulation is needed to cover or to create a protective layer for the electronic components which have been assembled on the circuit on flexible substrate. PDMS is chosen because it has higher flexibility and required a low curing temperature. It was carried out by using the 184 Silicone Elastomer; PDMS (Sylgard 184, Dow Corning). The PDMS mixture is prepared by mixing the Elastomer base with the curing agent according to a 10:1 ratio (by weight) in a Petri dish. After sufficient stirring, to ensure the reaction between the mixture and no air bubbles trapped within, the mixture is let rest for 30 minutes. After 30 minutes, the mixture is poured on the flexible circuit which has been placed in a mold. Then, it has been cured at $100^{\circ} \mathrm{C}$ in an oven for 35 minutes. There are several choices of curing temperature such as $100^{\circ} \mathrm{C}$ for 35 minutes, $125^{\circ} \mathrm{C}$ for 20 minutes or even at $150^{\circ} \mathrm{C}$ for 10 minutes. According to mechanical characterization that have been done by Johnston [15], with $100^{\circ} \mathrm{C}$ curing time was able to give a high ultimate tensile strength of $6.25 \mathrm{MPa}$.

\subsection{Data acquisition}

There are ten subjects involved in the data acquisition in this study. There is no subjects' gender restriction. Subjects' age is around 19 to 22 years old. In each set of activity, subjects are needed to rest for 5 minutes before running activity. Then, subjects must run on a treadmill with $0^{\circ}$ of inclination at $1 \mathrm{~km}$ per hour $(\mathrm{km} / \mathrm{h}), 0^{\circ}$ at $2 \mathrm{~km}$ per hour and $0^{\circ}$ at $3 \mathrm{~km}$ per hour. Next set of activity is running on the treadmill with $3^{\circ}$ of inclination at $1 \mathrm{~km}$ per hour, $3^{\circ}$ at $\mathrm{km}$ per hour and $3^{\circ}$ at $3 \mathrm{~km}$ per hour. Each activity is done for one minute for data recording. For optimal data acquisition by using the fabricated ECG circuit, the device is placed diagonally, following Lead III electrode position of Einthoven's Triangle [16]. Positive electrode (labelled as SEP pin) of the device is connected to torso (the trunk of the human body), while negative 
electrode (labelled as SEN pin) is connected to the area nearer left arm (LA). For a clearer configuration of electrode's position, please refer to Figure 2. The ECG device was turned on and the Bluetooth was connected to the smartphone accordingly. Once the Bluetooth of the ECG device and phone are both paired, it is ready to be used. Android application of ECG is opened and device Bluetooth name is chosen. Then, the phone started to display the subjects' ECG signals and data file is created and saved at the same time inside the smartphone internal memory.

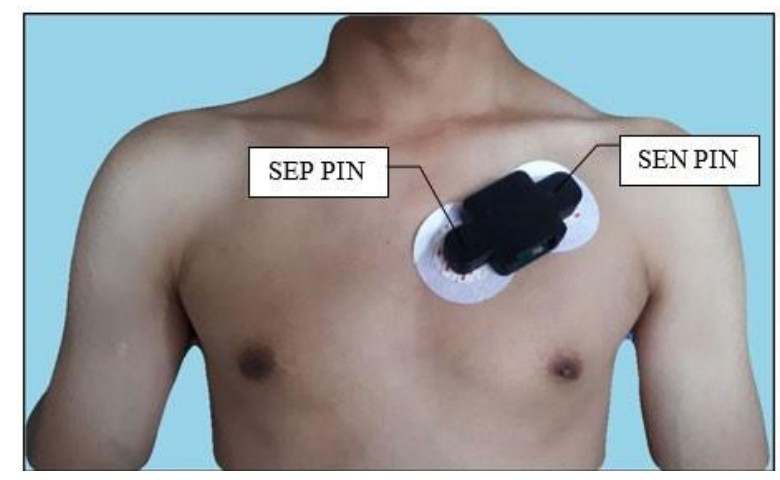

Figure 2. Eletrodes' position on subject

\section{RESULTS AND DISCUSSION}

\subsection{ECG circuit prototype and validation}

In this subsection, ECG circuit validation process was discussed. Figure 3 shows the circuit design. Figure 3(a) shows the position of each component used for the design. The circuit is designed using EAGLE software with a dimension of $76 \mathrm{~mm} \times 45 \mathrm{~mm}$. Figure 3(b) shows the prototype of ECG circuit which has been fabricated on PET substrate. All components on the substrate have been encapsulated with PDMS layer. Functional and electrical testing was done to ensure both circuits could provide an optimum performance and quality before being test with a human subject. System used for validation process is shown as in Figure 4. The circuits are connected to ECG patient's simulator from Fluke ProSim2 Vital Sign Simulator to generate the ECG signals. Normal ECH rhythm was chosen for the circuit testing. Connections between ECG simulator and ECG circuit are shown in Figure 4(a). Positive input of the circuit (labelled as SEP pin) is connected to Right Leg (RL) and negative input of the circuit (labelled as SEN pin) is connected to Left Arm (LA) of the patient simulator according to Lead III of Einthoven's Triangle theory. ECG circuit is supplied with a 3.7 V Lithium-Polymer (LiPo) battery. For ECG signal display and data collection, an ECG android application [17] as in Figure 4(b) was used to receive and display ECG data which has been transmitted from the developed ECG device. Then, the data are saved in the smartphones' memory in .txt format. These data were used for further analysis.

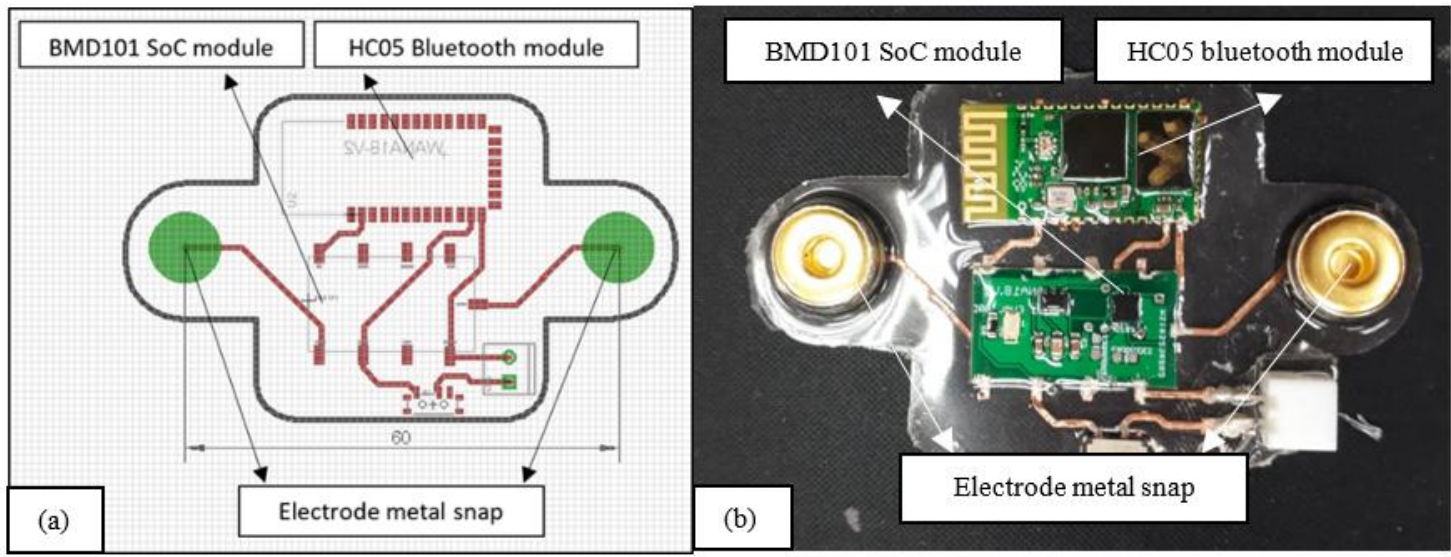

Figure 3. ECG circuit design, (a) Board layout from EAGLE software, (b) Prototype of ECG circuit on PET substrate 


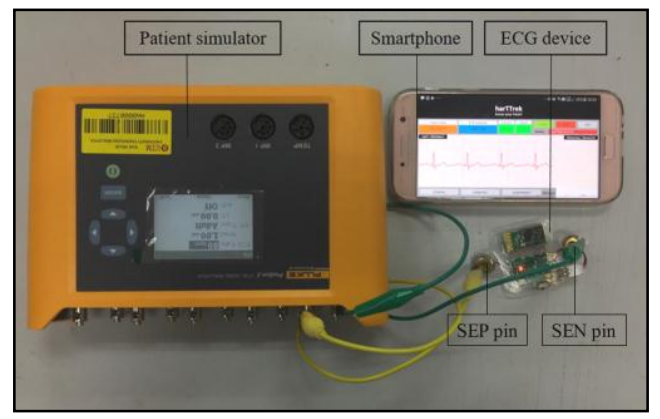

(a)

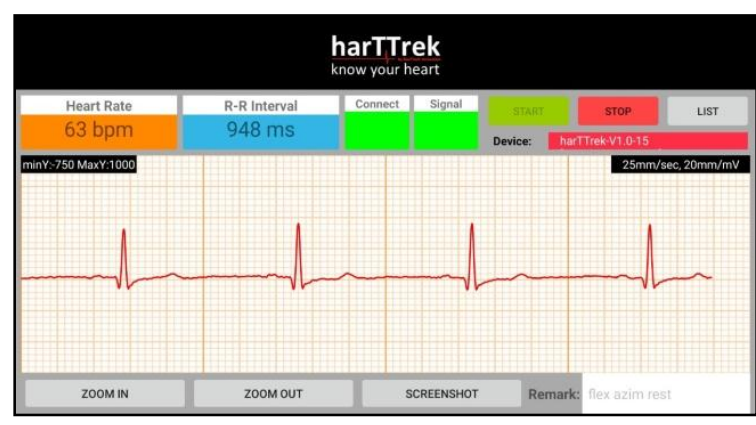

(b)

Figure 4. Validation process system, (a) Instrument setup for ECG circuit validation, (b) ECG signal displayed on smartphone for validation process using patient simulator

\subsection{ECG data analysis}

Previously, infinite impulse response (IIR) filter [18], finite impulse response (FIR) filter [19], adaptive filter [20, 21], moving average filter [22], Butterworth filter [23] have been used widely in ECG signal processing. Least mean square (LMS) adaptive filter is the most widely used in filtering ECG. But this method is not able to capture the rapidly varying non- stationary signal. However, in the last two decades, wavelet transform (WT) has been known among the researcher because its ability in analyzing nonstationary and Pseudo periodic ECG signal. In addition, WT filter does not require a reference model and has a higher accuracy according to a review paper [24]. There are a lot of family and scope of WT to be used in processing the ECG signal such as Daubechies, Coiflets, Biorthogonal and Symlets. Based on previous research [25], they found that Symlets family provides the best performance according to the SNR and (percent root mean square distortion) PRD value measured in the research. Thus, Symlets family of WT is used in this study to process and filter the ECG signal acquired during the experiment. In short, the first step in producing a wavelet denoising is to choose a wavelet function (first parameter) to be used in signal decomposition. Different types of wavelet are available of which each type has different sub-types. The second step is to choose a suitable decomposition level (second parameter). It must be selected with a family wavelet function. The third step is the selection of threshold value (third parameter) to be applied in the wavelet domain. Thus, wavelet basis function used in this study is Symlets wavelet and decomposition level used is level three. Threshold value used in this study is referred to soft threshold method.

Figure 5 shows the decomposition of the ECG signal using sym20 wavelet at level 3 . Based on the figure, $\mathrm{S}$ is referred to the mother wavelet or raw signal, $a a_{3}$ is the third approximation signal from the output of the down sampled of second LPF, $d d 3$ is down sampled output of third HPF, dd2 is down sampled output of second HPF and $d d 1$ is down sampled output of first HPF.



Figure 5. Decomposition of ECG signal using sym20 
Since the data have been filtered using WT tool as discussed in previous section, the differentiation between signal and noise can be done. The SNR for each subject and each activity is calculated. Based on the activities described in section 2.2, there are seven activities done by each subject. For each activity's, SNR mean values were calculated and summarized as in Table 1. From the SNR values of all activities, average SNR value calculated for flexible circuit is (32.10) in ratio. These value then was compared to SNR value obtained by [25], which is (32.25) in ratio. This shows there is no great distinctive between SNR values of flexible circuit type from this study and the value of SNR from the previous study [19]. From this SNR comparison, it can be concluded that both types of ECG circuit signals qualities are comparable with the device used for ambulatory ECG recordings in hospital.

Table 1. SNR mean values for each activity

\begin{tabular}{cccc}
\hline Treadmill-cliff degree $\left(^{\circ}\right)$ and speed $(\mathrm{km} / \mathrm{h})$ & Subject $(\mathrm{N})$ & SNR $($ mean $)$ & Standard deviation \\
\hline $0^{\circ}, 0 \mathrm{~km} / \mathrm{h}($ Rest $)$ & 10 & 32.42 & 2.98 \\
$0^{\circ}, 1 \mathrm{~km} / \mathrm{h}$ & 10 & 31.56 & 3.25 \\
$0^{\circ}, 2 \mathrm{~km} / \mathrm{h}$ & 10 & 32.41 & 3.69 \\
$0^{\circ}, 3 \mathrm{~km} / \mathrm{h}$ & 10 & 32.08 & 3.59 \\
$3^{\circ}, 1 \mathrm{~km} / \mathrm{h}$ & 10 & 31.83 & 3.22 \\
$3^{\circ}, 2 \mathrm{~km} / \mathrm{h}$ & 10 & 32.31 & 2.75 \\
$3^{\circ}, 3 \mathrm{~km} / \mathrm{h}$ & 10 & 32.12 & 3.24 \\
\hline
\end{tabular}

\section{CONCLUSION}

A new method to fabricate ECG circuit has been introduced in this study. Thus, the quality of ECG signal is a major concern. Consequently, the quality of ECG signals acquired by using the fabricated flexible ECG circuit is compared to ECG database. Data collection was obtained from ten subjects of undergraduate students from Universiti Teknologi Malaysia. Total of seven activities (rest and run on treadmill with different speed and degree of inclination) were recorded for the data collection purposes. Average SNR value of ECG circuit on PET substrate is (32.10) which was compared with SNR value from a previous study (which used data from MIT-BIH Arrhythmia database of Physionet) is (32.25). There is no significance different could be observed between these two values thus support the method of using vinyl cutter machine to fabricate ECG circuit on flexible substrate shall be prominence for future study

\section{ACKNOWLEDGEMENTS}

The authors would like to thank Ministry of Higher Education Malaysia and Universiti Teknologi Malaysia for funding this study under Matching Grant vot No. Q.J130000.3001.01M13 and GUP Tier 2 vot No. Q.J130000.2651.17J08. Special thanks to Collaborative Research in Engineering, Science \& Technology Center (CREST) for their continuous support for this research and funding this study under industrial grant with UTM's vot. number R.J130000.7301.4B248

\section{REFERENCES}

[1] D. Jin, W. Lee, Y. Kim, Young-Sik K., J. Lyu, and Sang-Min K., "Flexible display panel and display apparatus including the flexible display panel," United States Patent, US 8,736,162,B2, 2014.

[2] K. Church, E. MacDonald, P. Clark, R. Taylor, D. Paul, K. Stone, M. Wilhelm, F. Medina, J. Lyke, and R. Wicke, "Printed electronic processes for flexible hybrid circuits and antennas," 2009 Flexible Electronics \& Displays Conference and Exhibition, pp. 1-7, 2009.

[3] Y. Kurokawa, T. Ikeda, M. Endo, H. Dembo, D. Kawae, T. Inoue, M. Kozuma, D. Ohgarane, S. Saito, K. Dairiki, H. Takahashi, Y. Shionoiri, T. Atsumi, T. Osada, K. Takahashi, T. Matsuzaki, H. Takashina, Y. Yamashita, and S. Yamazaki, "UHF RFCPUs on flexible and glass substrates for secure RFID systems," IEEE Journal of SolidState Circuits, vol. 43, no. 1, pp. 292-299, Jan. 2008.

[4] M. A. Fonseca, M. G. Allen, J. Kroh, and J. White, "Flexible wireless passive pressure sensors for biomedical applications," Tech. Dig Solid-State Sensors, Actuators and Microsystems Workshop 2006, no. 1, pp. 37-42, 2006.

[5] Y. L. Tai and Z. G. Yang. "Fabrication of paper-based conductive patterns for flexible electronics by directwriting," Journal of Materials Chemistry, vol. 21, no. 16, pp. 5938-5943, Mar. 2011.

[6] J. Suikkola, T. Björninen, M. Mosallaei, T. Kankkunen, P. Iso-Ketola, L. Ukkonen, J. Vanhala, and M. Mäntysalo, "Screen-printing fabrication and characterization of stretchable electronics," Scientific Reports, vol. 6, pp. 1-8, 2016.

[7] A. Eshkeiti, "Novel stretchable printed wearable sensor for monitoring body movement, temperature and electrocardiogram, along with the readout circuit," Ph.D Dissertation, Electrical and Computer Engineering Department, Western Michigan University, Aug. 2015.

[8] S. Busato, A. Belloli, and P. Ermanni, "Inkjet printing of palladium catalyst patterns on polyimide film for electroless copper plating," Sensors and Actuators B: Chemical, vol. 123, no. 2, pp. 840-846, 2007. 
[9] S. Nagels, R. Ramakers, K. Luyten, and W. Deferme, "Silicone devices: A scalable DIY approach for fabricating self-contained multi-layered soft circuits using microfluidics," in Proceedings of the 2018 CHI Conference on Human Factors in Computing Systems, no. 188, pp. 1-13, 2018.

[10] W. Jensen, A. Colley, J. Häkkilä, C. Pinheiro, and M. Löchtefeld, "TransPrint: A method for fabricating flexible transparent free-form displays," Advances in Human-Computer Interactation, vol. 2019, pp. 1-14, 2019.

[11] N. S. Sahar, N. A. Abdul-Kadir and F. K. Che Harun, "Flexible wireless ECG circuit fabrication technique," 2019 International Biomedical Instrumentation and Technology Conference (IBITeC), pp. 21-25, 2019.

[12] N. S. Sahar, N. A. Abdul-Kadir, W. H. Chan, S. L. M. Eileen, F. K. C. Harun. "Wireless ECG circuit on flexible material: A preliminary study," J. of Telecom., Electronic and Comp. Eng., vol. 10, no. 1-17, pp. 43-46, 2018.

[13] N. A. Abdul-Kadir, N. S. Sahar, W. H. Chan and F. K. C. Harun, "A portable WiFi ECG," 2018 IEEE 38th International Electronics Manufacturing Technology Conference (IEMT), pp. 1-4, 2018.

[14] H. A. Andersson, A. Manuilskiy, S. Haller, M. Hummelgård, J. Sidén, C. Hummelgård, H. Olin, and Hans-Erik N., "Assembling surface mounted components on ink-jet printed double sided paper circuit board," Nanotechnology, vol. 25, no. 9, pp.1-9, 2014.

[15] I. D. Johnston, D. K. McCluskey, C. K. L. Tan, and M. C. Tracey, "Mechanical characterization of bulk Sylgard 184 for microfluidics and microengineering," J. of Micromech. and Microeng., vol. 24, no. 3, pp. 1-7, 2014.

[16] Rui Zou, Chung-Lieh Hung, Chi Ln Lo, C. Yang, and W. Zhang, "Evaluation of the accuracy of ECG captured by CardioChip through comparison of ECG recording to a standard 12-lead ECG recording device," 2016 IEEE 25th International Symposium on Industrial Electronics (ISIE), pp. 1094-1098, 2016.

[17] FlexTTech Innovation Sdn. Bhd., “2018062845-Submit for trademark: Perbadanan harta intelek Malaysia,” 2019.

[18] L. Gu, N. Zhou, and H. Wu, "Application of interference canceller in bioelectricity signal disposing," Procedia Environmental Sciences, vol. 10, pp. 814-819, 2011.

[19] G. Kadam and P. C. Bhaskar, "Reduction of power line interference in ECG signal using FIR filter," International Journal of Computational Engineering Research, vol. 2, no. 2, pp.314-319, 2012.

[20] N. V. Thakor and Y. S. Zhu, "Application of adaptive filtering to ECG analysis: Noise cancelation and arrythmia detection," IEEE Transactions on Biomedical Engineering, vol. 38, no. 8, pp. 785-794, 1991

[21] S-H. Liu, "Motion artifact reduction in electrocardiogram using adaptive filter," J. Med. Biol. Eng., vol. 31, no. 1, pp. 67-72, 2011.

[22] M. A. Othman and N. M. Safri, "Characterization of ventricular arrhythmias using a semantic mining algorithm," Journal of Mechanics in Medicine and Biology, vol.12, no. 3, pp. 1250049-1-11, 2012.

[23] N. A. Abdul-Kadir, M. A. Othman, and N. M. Safri, "ECG features extraction using second-order dynamic system and regeneration using hybrid recurrent network," 2016 IEEE Int. Conf. on Signal Processing, Communications and Computing (ICSPCC), pp. 1-5, 2016.

[24] S. Thalkar and D. Upasani, "Various techniques for removal of power line interference from ECG signal," Int. J. Sci. Eng. Res., vol. 4, no. 12, pp. 12-23, 2013.

[25] R. Khanam, and S. N. Ahmad, "Selection of wavelets for evaluating SNR, PRD and CR of ECG signal," International Journal of Engineering Science and Innovative Technology, vol. 2, no. 1, pp.112-119, 2013.

\section{BIOGRAPHIES OF AUTHORS}

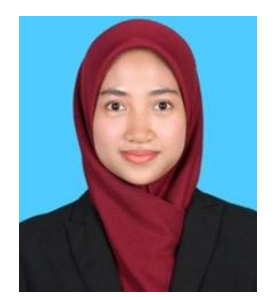

Noor Syazwana Sahar received her Bachelor's Degree in Electrical (Medical Electronics) Engineering from Universiti Teknologi Malaysia in 2014. Previously, she worked as Product Engineer in Freescale Semiconductor, Petaling Jaya. Currently, Syazwana pursuing her Master of Philosophy study in Biomedical Engineering at Universiti Teknologi Malaysia. Her research area related to bio-signal processing of ECG and electronic circuit.

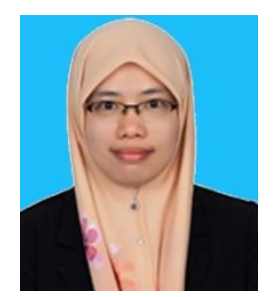

Nurul Ashikin Abdul-Kadir serves at Universiti Teknologi Malaysia in the field of Electrical and Electronic Engineering as Senior Lecturer since 2019. She received her master and phD degree in Electrical Engineering and her bachelor degree in Electrical Engineering (Medical Electronics). Her research interest is related to electronic circuit, bio-signal processing (ECG, EEG, EMG), embedded electronics and pattern recognition.

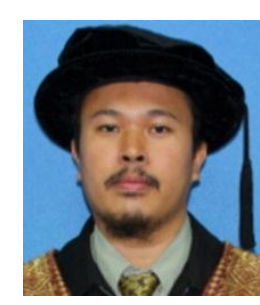

Fauzan Khairi Che Harun received his BEng degree in Electrical-Electronics Engineering from Universiti Teknologi Malaysia in 2003. He then further his study in the area of biomedical engineering in University of Warwick where he was awarded with MSc in Advanced Electronic (2005) and $\mathrm{PhD}$ in Engineering (2010). His research interests are mainly but not restricted to biomedical electronics, Biological Inspired Micro-system and Electronic Nose System. Currently he is a senior lecturer at the School of Electrical Engineering, UTM. 